\title{
Interrogation of Multimeric DNA Amplification Products by Competitive Primer Extension Using Bst DNA Polymerase (Large Fragment)
}

BioTechniques 31:1122-1129 (November 2001)

\author{
J. Voisey, G.J. Hafner, \\ C.P. Morris, A. van Daal, and \\ P.M. Giffard \\ CRC for Diagnostics, Brisbane, \\ Australia
}

\section{INTRODUCTION}

It is now well established that DNA molecules composed of tandem repeats mInitallyay be amplified exponentially under isothermal conditions using strongly strand-displacing DNA polymerases and two primers, each specific for one of the strands $(10,16,18)$. In most previous reports, the source of the multimeric substrate molecule has been rolling circle replication of a synthetic single-stranded circular template of approximately 100 bases, and the production and subsequent amplification of the linear multimer has been termed cascade rolling circle amplification (CRCA). It has also recently been found that linear molecules may be amplified and multimerized under similar reaction conditions (6).

In an endeavor to adapt CRCA to routine diagnostic applications requiring single nucleotide specificity, we have devised a microplate-based strategy for amplification, amplified material detection, and SNP discrimination therein that incorporates a long non-allele-specific amplification step and a short com petitively primed allele-specific amplification step. This method makes use of an immobilized primer and a solution phase primer and is similar to the solidphase PCRs described by Rasmussen et al. (15) and Morris and Harris (11). The reaction is also similar to the solidphase, single-primer, RCA-based procedure described by Hatch et al. (7), with the major differences that the allele-specific primers are in the solution phase and two steps of exponential amplification are used.

\section{MATERIALS AND METHODS}

\section{Oligonucleotide Synthesis}

Oligonucleotides were supplied by Invitrogen (Mulgrave, Victoria, Australia) or by GeneWorks (Adelaide, South Australia, Australia). Oligonucleotides used as padlocks, and targets were gel purified to homogeneity by the suppliers. All other oligonucleotides were supplied as desalted preparations.

\section{Oligonucleotide Sequences Derived from Chlamydia}

Padlocks were designed essentially as described by Nillson et al. (13). They were based on the reported sequences for the groEL genes from Chlamydia trachomatis and Chlamydia pneumoniae $(2,9)$.

The Chlamydia-specific padlock was 5'-GCAGGTAAAGAAGGCGCCGCGGTGAGCTATATGGGGACTATGAATTTGCTCCATTAAAGCAAAT TG C-3'. The bases designed to hybridize to either or both of the targets are underlined, the bases designed to hybridize to the immobilized common primer FVComT are italicized, and the region identical to the allele-specific primers (minus 4 bases at the $3^{\prime}$ ends, which are variable and terminate in the spacers) is in bold.

The $C$. pneumoniae-derived target was 5'-ATAGCGCCTTCTTTACCTG CGTTACTTCGAATTTGCTTTAATGGAGCTG-3'. The bases designed to hybridize to the padlock are underlined. The $C$. pneumonia-specific spacer was 5'-AAGTAAC-3'. The T was biotin- 
ylated, but this was not used as a capture tag in any experiments. The $C$. trachomatis-derived target was $5^{\prime}$-ATAGCACCTTCTTTTCCTGCGTTTGC ACGAATTTGTTTCAAAGGAGCGG-3'. The bases designed to hybridize to the padlock are underlined. The $C$. trachomatis-specific spacer was 5'-TG CAAAC-3'. The T was biotinylated, but this was not used as a capture tag in any experiments.

The $C$. pneumoniae- and $C$. trachomatis-specific amplification primers were each designed to terminate within the corresponding spacer sequences. They were of sequence 5'-CCATTAAAGCAAATTGCAAG-3' and $5^{\prime}$-CCATTAAAGCAAATTGCTG C A-3', respectively, and labeled with 6-carboxy fluorescein (FAM) at the $5^{\prime}$ $\mathrm{C}$ residues. The non-allele-specific primer (FVComT) was immobilized to the microwell wall. It was designed to be complementary to the padlock and to incorporate a poly- $\mathrm{T}$ spacer sequence at the $5^{\prime}$ end. Its sequence was 5'-TTTTTTTTTTGTCCCCATATAG CTCACCG-3'.

\section{Oligonucleotide Sequences Derived from the Factor V Leiden Mutation Site (1)}

The padlock was 5'-AGGAATA CAGGTATTTTGTCCTTGC GCGGTGAGCTATATGGGGACTATGAATTTCTAATAGGACTACTTCTAATCTGT-

AAGAG 3 '. The regions that hybridize to the target are underlined, and the region complementary to the common primer FVComT is italicized. The spacer specific for the wild-type sequence was 5'-CAGATCCCTGGACAGGCG$3^{\prime}$. The spacer specific for the mutant sequence was 5'-CAGATCCCTGGACAGGCA-3'. The FAM-labeled, allele-specific primers were of the same sequences as the spacers, except that sub-terminal mismatches are incorporated. They were $5^{\prime}$-CAGATCCCTGG ACAGACG-3' (wild-type) and 5'-C AGATCCCTGGACAGACA-3' (mutant) and with labels at the $5^{\prime}$ terminal $C$ residues. The unlabeled primers were the same sequence but without the $5^{\prime}$ terminal CA residues. The non-allelespecific immobilized primer used was

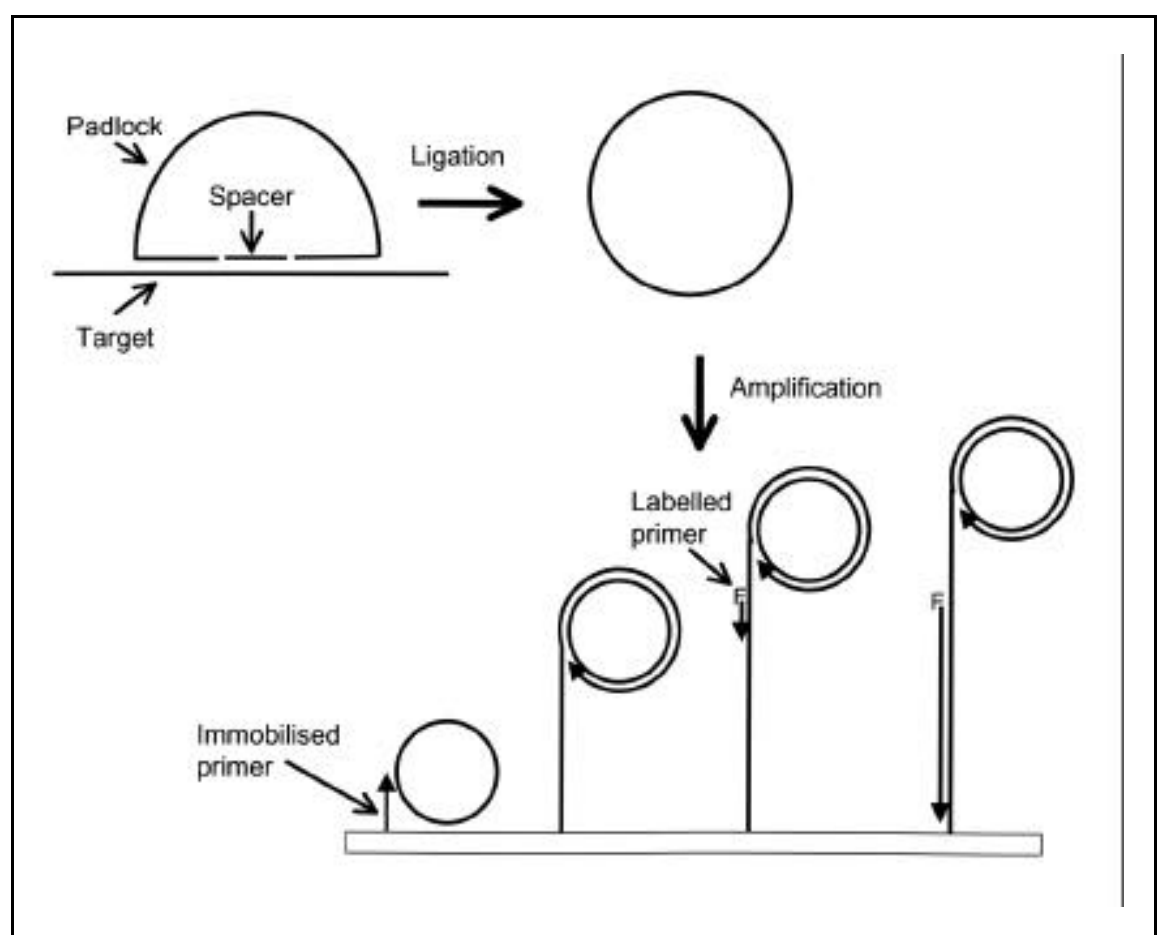

Figure 1. The essential elements of solid-phase CRCA. Target- and ligase-mediated padlock circularization precedes CRCA with one immobilized primer and one or more labeled solution-phase primers. During the exponential phase of the reaction, it is likely that the extended labeled primer is displaced by subsequent priming events and then serves as a template and/or primer for additional extension reactions, thus yielding a complex network of linear double-stranded concatemers. Therefore, the labeled primer may frequently prime on a linear molecule that is not directly connected to the circular template.

FVComT, the same as for the Chlamydia-derived system.

\section{Binding of FVComT to Microwells}

NUNCTM NucleoLink Strips (obtained from Medos Company, Mt. Waverley, Victoria, Australia) were used throughout. To each well was added 100 $\mu \mathrm{L} 100$ nM FVComT, 10 mM 1-ethyl-3(3-dimethylaminopropyl)-carbodiimide, $10 \mathrm{mM}$ 1-methyl-imidiazole. The wells were sealed with cling-film and incubated at $50^{\circ} \mathrm{C}$ overnight. The solution was then removed, and the wells were washed three times with $100 \mathrm{mM}$ Tris$\mathrm{HCl}, \mathrm{pH} 7.5,150 \mathrm{mM} \mathrm{NaCl}, 0.1 \%$ Tween ${ }^{\circledR} 20$ (TBST), soaked for $5 \mathrm{~min}$, and then washed three more times with the same buffer. Finally, the wells were washed once with deionized water and stored at $4{ }^{\circ} \mathrm{C}$.

\section{Padlock Circularization}

Target DNA was mixed with 1 pmol padlock, 1 pmol spacer molecule, and 5 U Ampligase ${ }^{\circledR}$ DNA ligase in Ampligase buffer (both from Epicentre Technologies, Austral Scientific, Gymea, NSW, Australia) in a total volume of 50 $\mu \mathrm{L}$. The mixture was heated to $94^{\circ} \mathrm{C}$ for $3 \mathrm{~min}$ and then incubated at $60^{\circ} \mathrm{C}$ for $20 \mathrm{~min}$.

\section{Primary and Single-Step, Amplification Reactions}

Each reaction contained $1 \mu \mathrm{L}$ ligation reaction products, $10 \mathrm{mM}$ dNTPs (Roche Molecular Biochemicals, Indianapolis, IN, USA), $166 \mathrm{nM}$ solution phase primers made up to a final volume of $60 \mu \mathrm{L}$ in thermopolymerase buffer (New England Biolabs; Genesearch, Arundel, Queensland, Australia). The mixture was heated to $94^{\circ} \mathrm{C}$ for $3 \mathrm{~min}$, then $4 \mathrm{U}$ Bst DNA polymerase (large fragment) (New England Biolabs) were added, and the reaction was incubated for $90-120 \mathrm{~min}$ at $60^{\circ} \mathrm{C}$.

\section{Secondary Amplification Reactions}

These were carried out in Nunc NucleoLink Strips coated with primer FV ComT as described above. The reaction conditions were the same as for the primary amplification reactions, except 
that the solution-phase primer were added to $333 \mathrm{nM}$, the substrates were reaction products from primary amplification reactions, and the incubation was for $10 \mathrm{~min}$ at $60^{\circ} \mathrm{C}$.

When the primary amplification was carried out in a microcentrifuge tube, $10 \mu \mathrm{L}$ of the primary amplification products were added to the secondary amplification reaction. When the primary amplification was carried out in a microwell, the allele-specific primers and a further $4 \mathrm{U} B s t$ DNA polymerase (large fragment) were added to the primary amplification products.

\section{Quantitation of Immobilized FAM-Labeled Primer}

The solution-phase amplification products were removed from the wells, which were then washed three times with TBST at room temperature, twice with TBST at $80^{\circ} \mathrm{C}$ (incorporating a 2-min incubation in the presence of each aliquot of TBST), and then once more with TBST at room temperature. The final wash solution was removed, and the wells were blocked with $50 \mu \mathrm{L}$ $10 \mathrm{mg} / \mathrm{mL}$ BSA for $1 \mathrm{~h}$ and then washed three times with TBST. Diluted anti-fluorescein antibody-alkaline phosphatase conjugate $(50 \mu \mathrm{L}, 1: 3000$; Roche Molecular Biochemicals) was added to the wells, which were then incubated at room temperature for 30 min. The antibody solution was removed, and the wells were washed six times with TBST at room temperature and then twice with $100 \mathrm{mM}$ Tris- $\mathrm{HCl}$, $\mathrm{pH} 7.5,150 \mathrm{mM} \mathrm{NaCl}$ at room temperature. Para-nitrophenylene phosphate (PNPP) (50 $\mu \mathrm{L}$; Sigma) solution (1 $\mathrm{mg} / \mathrm{mL}$ in the buffer provided by the manufacturer) was then added to the wells, which were incubated in the dark for $30 \mathrm{~min}$. The absorbances were then read at $405 \mathrm{~nm}$.

\section{RESULTS}

\section{Adaptation of CRCA to the Solid Phase}

The solid-phase CRCA method is illustrated in Figure 1. Initial experiments to develop solid-phase CRCA reactions made use of synthetic targets derived from the $h s p 60$ genes of $C$. trachomatis and $C$. pneumoniae. A synthetic padlock was designed to be able to anneal to both targets leaving a 6base gap between the padlock ends. Species-specific spacer molecules were designed to be able to anneal within

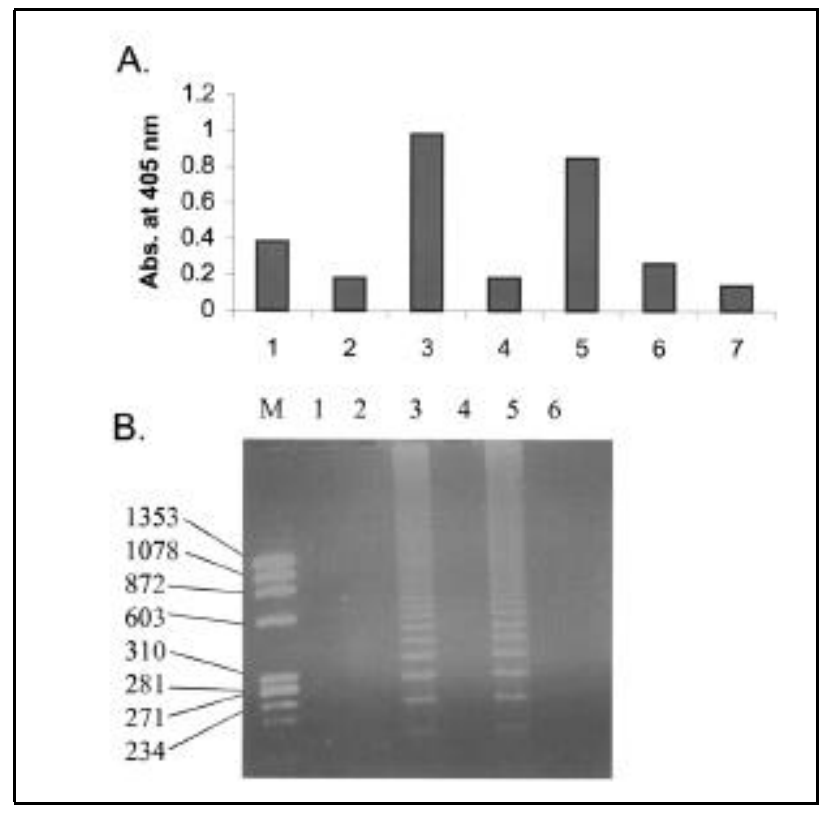

Figure 2. SPCRCA using synthetic Chlamydia-derived targets, padlock, spacers, and primers. The ligation reactions contained 50 pmol target unless otherwise indicated. The concentrations of other reagents were as stated in the Materials and Methods section. (A) Absorbance readings after 60 min color development. Lane 1, negative control 1, no target, $C$. pneumoniae-specific spacer and primer; lane 2, negative control $2, C$. pneumoniae-specific target, spacer, and primer, no ligase; lane 3, C. pneumoniae-specific target, spacer, and primer; lane 4, C. pneumoniae-specific target, C. trachomatis-specific spacer and primer; lane 5, C. trachomatisspecific target, spacer, and primer; lane 6, C. trachomatis-specific target, C. pneumoniae-specific spacer and primer; and lane 7, negative control 3, color development reagents only. (B) Electrophoresis in $2 \%$ agarose of the solution-phase material from panel A. Lane M, molecular weight marker (base pairs indicated) (Roche Molecular Biochemicals). Lanes 1-7 are the same as for panel A.

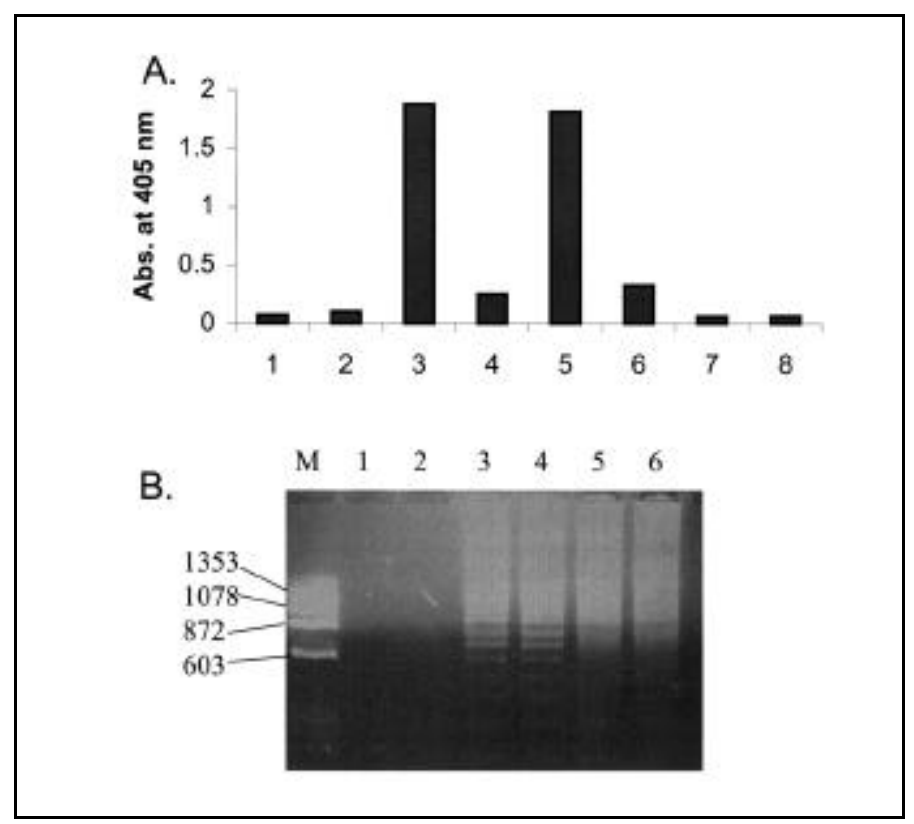

Figure 3. Post-amplification competitively primed SPCRCA using Factor V Leiden mutation-derived target, padlock, spacers, and primers. The $1 \mathrm{pmol}$ synthetic target was used. The first nonspecific amplification was carried out in microcentrifuge tubes. (A) Absorbance readings after 30 min color development. Lane 1 , no target, second amplification reaction only, mutant-specific spacer, labeled mutant-specific primer, unlabeled wild-type-specific primer; lane 2, the same as for lane 1, but both amplification reactions were carried out; lane 3, wild-type-specific target and spacer for first amplification, labeled wild-type-specific primer and unlabeled mutant specific primers in second amplification; lane 4, wild-type target and spacer for first amplification, labeled mutant-specific and unlabeled wild-type-specific primers in second amplification; lane 5, mutant-specific target and spacer in first amplification, labeled mutant-specific and unlabeled wild-type-specific primers in second amplification; lane 6, mutant-specific target and spacer in first am plification, labeled wild-type-specific primer and unlabeled mutant-specific primers in second amplification; and lanes 7 and 8, color development reagents only. (B) Electrophoresis of the solution-phase material from panel A. Lane M, molecular weight markers (base pairs indicated). Lanes 1-6 are the same as for panel A. 
this gap leaving single-strand breaks. Thus, ligase-mediated padlock circularization would be expected to occur only when the spacer sequence was complementary with the target, and the circularized padlock molecule would be expected to incorporate the spacer.

Figure 2 shows that detectable am plified material was synthesized only when the spacer and target were homologous. It can also be seen that the presence of detectable amplified material corresponded to high absorbance readings from the assays. We have termed this reaction solid-phase cascade rolling circle amplification (SPCRCA).

\section{Post-Amplification Competitive Priming Provides Single-Nucleotide Specificity}

To be able to interrogate single-nucleotide polymorphism (SNPs) in a target molecule, specificity can be introduced at either the padlock circularization or the amplification step. Here, we have addressed the problem of making the amplification step allele specific.

The most commonly used strategy for carrying out allele-specific amplification has been termed ARMS (12) or ASPCR (17). This makes use of a primer pair in which one of the amplification primers is specific for one of the alleles.

Initially, we attempted a strategy analogous to ASPCR/ARMS. Single-stranded circular templates differing at single positions were generated from synthetic targets, padlocks, and allele-specific spacer molecules. To ensure that the template molecules were homogeneous, the spacer used was always a perfect match for the target. Despite repeated attempts, we were unable to make the amplification reaction reproducibly allele specific [i.e., the $B s t$ DNA polymerase (large fragment) was

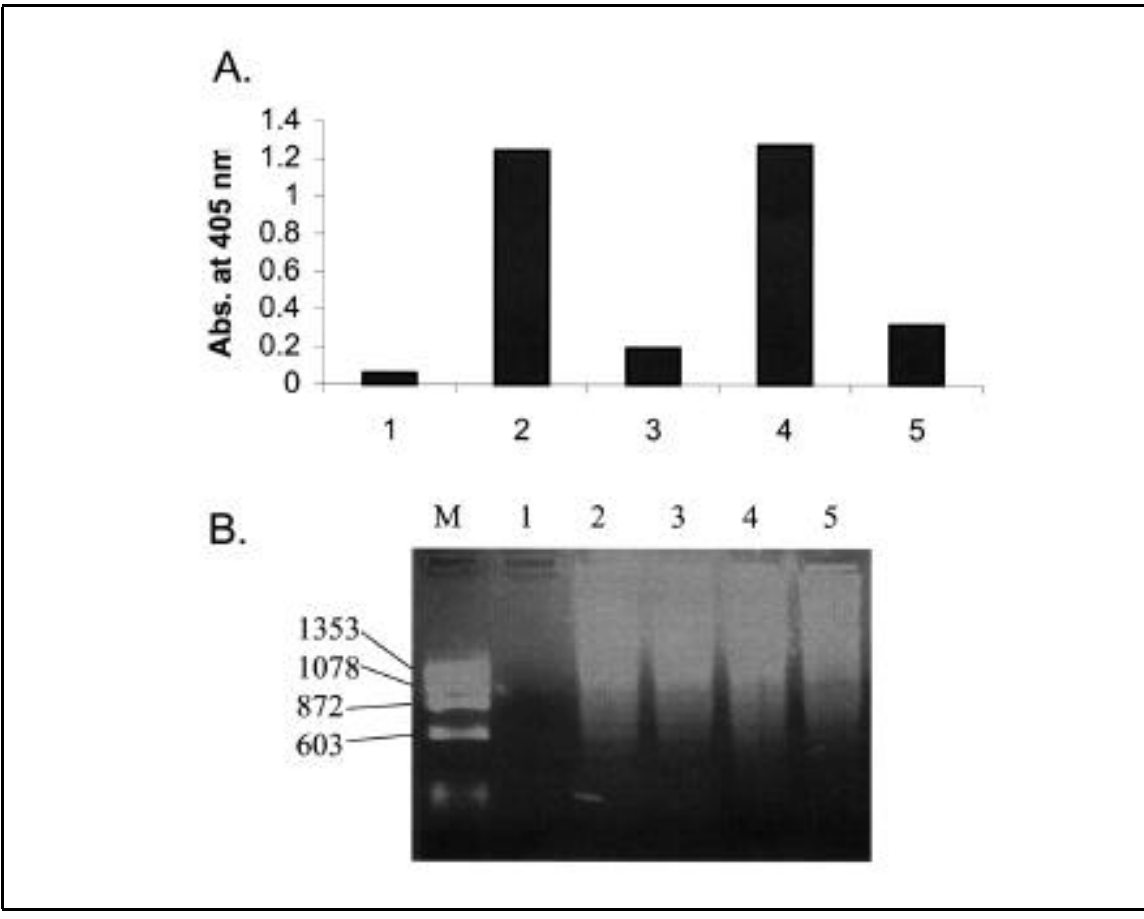

Figure 4. Post-amplification competitively primed SPCRCA using Factor V Leiden mutation-derived target, padlock, spacers, and primers. Human genomic DNA (500 ng) was used as the target. The first and second amplification reactions were carried out in the same microwells. (A) Absorbance readings after 30 min color development. Lane 1, no target, mutant-specific spacer, labeled mutant-specific primer, unlabeled wild-type-specific primer. Both amplification reactions were carried out; lane 2 , homozygous wild-type target, wild-type spacer, labeled wild-type-specific primer, unlabeled mutant-specific primer; lane 3, homozygous wild-type-specific target, wild-type-specific spacer, labeled mutantspecific primer, unlabeled wild-type-specific primer; lane 4, heterozygous mutant target, mutant-specific spacer, labeled mutant-specific primer, unlabeled wild-type-specific primer; lane 5, heterozygous mutant target, mutant-specific spacer, labeled wild-type-specific primer, unlabeled mutant-specific primer. (B) Electrophoresis of the solution-phase material from panel A. Lane M, molecular weight markers (base pairs indicated). Lanes 1-5 are the same as for panel A.

prone to extension from mismatched $3^{\prime}$ ends (data not shown)]. It was concluded that, in these reactions, Bst DNA polymerase (large fragment) extended from mismatched primers at a high enough frequency to preclude the use of this method.

To circumvent this, a competitive priming strategy was adopted. This differs from ASPCR in that two allelespecific primers are included in the reaction, and the amplified material is analyzed to determine which primer had been incorporated (3-5). Although competitive priming complicates gelbased assays, it does not significantly complicate SPCRCA because the readout from the reaction is from the labeled allele-specific primer. Therefore, the experimental system made use of pairs of reactions in which one allelespecific primer was labeled and the other was not labeled. This provided some improvement in specificity, although the robustness and reproducibility were still unsatisfactory (data not shown).

It was recognized that, in a competitively primed amplification reaction, there is a direct relationship between the degree of amplification and the proportion of total amplified material that is a product of primer misincorporation. This is because, as amplification proceeds, any products of mispriming are replicated while new mispriming events occur. Therefore, it was predicted that reducing the extent of the allelespecific amplification would increase its specificity.

This was found to be the case. If the circular template was first amplified by CRCA in a non-allele-specific manner, and the amplified material was then interrogated by competitive priming for 10 min under similar reaction conditions, then the relative incorporation of the allele-specific primers was in accordance with the allele present in the circular template. Initially, the first amplification was performed in a microcentrifuge tube, and the interrogation of the amplified material was performed in a microwell. High absorbance readings were obtained from the positive reactions, indicating that the 10-min amplified material detection step was sufficient for significant priming from the immobilized primer (Figure 3). Similar results were obtained when both amplification reac- 
tions were carried out in the same microwell and a genomic target was used (Figure 4). The similar quantity of solution-phase amplified material in the different reactions illustrates that the specificity observed was due to differential primer incorporation rather than differential degrees of amplification. Allele specificity was found to be robust upon repetition of the reactions, and careful optimization of primer concentration was not necessary.

\section{DISCUSSION}

Optimization of ASPCR can sometimes be difficult because of the measurable ability of Taq DNA polymerase to extend from primers mismatched at the 3 ' end (8), the exponential replication of any mispriming products, and the possibility that a positive reaction may enter a plateau phase while the negative reac- tion is in fully exponential phase. Although we have not quantified the ability of Bst DNA polymerase (large fragment) to extend from mismatched primers, the difficulties we have experienced in optimizing a Bst DNA polymerase (large fragment)-based method analogous to ASPCR suggests that its inherent ability to do this is high.

The adoption of a competitive prim ing strategy would not be expected to reduce the incidence of extension from mismatched primers. Rather, the production of amplified material by the matched and common primers increases the possibilities for mispriming as the reaction proceeds. Despite this, competitive priming can circumvent problems associated with the ASPCRlike methods because the reactions would be expected to terminate at the same time whether labeled or unlabelled primer is incorporated into the amplified material.
With this system, it was found that the ability of Bst DNA polymerase (large fragment) to misprime was sufficiently high that the adoption of a postamplification interrogation strategy was necessary to obtain robust single-nucleotide specificity. Although this increases the complexity of the procedure, a potentially useful variant of this strategy would be to carry out a single solution-phase multiplex amplification reaction and interrogate this in a num ber of microwells, each of which containing the same "common" primer. Alternatively, a primer array could be used in which each spot is composed of a target-specific but not allele-specific primer. The majority of reported array/primer extension-based primer SNP interrogation methods make use of im mobilized allele-specific primers (e.g., Reference 14). Our alternative strategy of using solution-phase, differentially labeled, allele-specific primers allows 
truly competitive priming reactions in which the amplification from perfectly matched primer-template sets takes place in every microwell or at every array location. The use of Bst DNA polymerase (large fragment) provides the potential for carrying this out isothermally and also incorporating more than one labeled primer molecule per immobilized primer molecule because of the multimeric nature of the amplified material.

For this method to be useful for genotyping, the genetic information must be transduced from the target to the padlock so that it can be amplified. We have tested both competitive ligation of spacer oligonucleotides and T7 DNA polymerase-mediated gap filling followed by ligation, and although these are successful when synthetic targets are used, in our hands they are unreliable with single-copy genes in human genomic samples (unpublished data). However, a sensitive gap-fill procedure has been previously reported (10), and it may be that the sensitivity of this reaction varies with different padlock sequences. If our strategy were to be combined with a reliable means of transducing the genetic information to the CRCA products, then a robust approach for isothermal SNP interrogation would result.

One unexpected result was that am plified material was present in the solution phase, even on those reactions in which an immobilized primer complementary to the padlock was present during the entire extent of the amplification reactions. This would be expected to yield reaction products in which all material complementary to the padlock (i.e., in the same sense as the immobilized primer) is attached to the microw ell wall. The likely explanation for this is that the very large molecules produced during amplification would be very easy to detach from the microwell wall through mechanical shearing.

In conclusion, the propensity of Bst DNA polymerase (large fragment) to carry out extension from mismatched primers may be circumvented by adopting a post-amplification competitive primer extension strategy. There is no reason why this approach could not be applied to other primer/polymerasebased amplification reactions.

\section{ACKNOWLEDGMENT}

This work was supported by the Australian Federal Government Program for Cooperative Research Centres.

\section{REFERENCES}

1.Bertina, R.M., B.P.C. Koeleman, T. Koster, F.R. Rosendaal, R.J. Driven, H. Ronde, P.A. van der Velden, and P.H. Reitsma. 1994. Mutation in blood coagulation factor associated with resistance to activated protein $\mathrm{C}$. Nature 369:64-67.

2.Cerrone, M.C., J.J. Ma, and R.S. Stephens. 1991. Cloning and sequence of the gene for heat shock protein 60 from Chlamydia trachomatis and immunological reactivity of the protein. Infect. Immun. 59:79-90.

3.Fauser, S. and B. Wissinger. 1997. Simultaneous detection of multiple point mutations using fluorescence coupled competitive primer extension. BioTechniques 22:964-968.

4.Germer, S. and R. Higuchi. 1999. Single tube genotyping without oligonucleotide probes. Genome Res. 9:72-88.

5.Gibbs, R.A., P-N. Nguyen, and C.T. Caskey. 1989. Detection of single DNA base differences by competitive oligonucleotide priming. Nucleic Acid Res. 17:2437-2448.

6.Hafner, G.J., I.C. Yang, L.C. Wolter, R. Stafford, and P.M. Giffard. 2001. Isothermal amplification and multimerization of DNA by $B s t$ DNA polymerase. BioTechniques 30:852860.

7.Hatch, A., T. Sano, J. Misasi, and C.L. Smith. 1999. Rolling circle amplification of DNA immobilized on solid surfaces and its application to multiplex mutation detection. Genet. Anal. 15:35-40.

8.Huang, M-M., N. Arnheim, and M.F. Goodman. 1992. Extension of base mispairs by Taq DNA polymerase: implications for single nucleotide discrimination in PCR. Nucleic Acid Res. 20:4567-4573.

9.Kikuta, L.C., M.H. Puolakkainen, C.-C. Kuo, and L.A. Campbell. 1991. Isolation and sequence analysis of the Chlamydia pneumoniae GroE operon. Infect. Immun. 59:4665-4669.

10.Lizardi, P.M., X. Huang, Z. Zhu, P. BrayWard, D.C. Thomas, and D.C. Ward. 1998. Mutation detection and single-molecule counting using isothermal rolling-circle replication. Nat. Genet. 19:225-232.

11.Morris, C.P. and R.J. Harris, inventors; Adelaide Children's Hospital and the University of South Australia, assignees. 1991. Solid phase amplification process. US patent No. 6017738.

12.Newton, C.R., A. Graham, L.E. Heptinsall, S.J. Powell, C. Summers, N. Kalshecker, J.C. Smith, and A.F. Markham. 1989. Analysis of any point mutation in DNA. The amplification refractory mutation system (ARMS). Nucleic Acid Res. 17:2503-2516.

13.Nillson, M., H. Malmgren, M. Samiotaki, M. Kwiatkowski, B.P. Chowdary, and U. Landegren. 1994. Padlock probes: circulariz- ing oligonucleotides for localized DNA detection. Science 265:2085-2088.

14.Pastinen, T., R. Raito, K. Lindroos, P. Tainola, L. Peltonen, and A.-C. Syvänen. 2000. A system for specific, high-throughput genotyping by allele-specific primer extension on microarrays. Genome Res. 10:1031-1042.

15.Rasmussen, S.R., H.B. Rasmussen, M.R. Larsen, R. Hoff-Jorgensen, and R.J. Cano. 1994. Combined polymerase chain reactionhybridization microplate assay used to detect bovine leukemia virus and Salmonella. Clin. Chem. 40:200-205.

16.Thomas, D.C., G.A. Nardone, and S.K. Randall. 1999. Amplification of padlock probes for DNA diagnostics by cascade rolling circle amplification or the polymerase chain reaction. Arch. Pathol. Lab. Med. 123:1170-1176.

17.Wu, D.Y., L. Ugozzoli, B.K. Pal, and R.B. Wallace. 1989. Allele-specific enzymatic am plification of $\beta$-globin genomic DNA for diagnosis of sickle-cell anemia. Proc. Natl. Acad. Sci. USA 86:2759-2760.

18.Zhang, D.Y., M. Brandwein, T.C. Hsuih, and H. Li. 1998. Amplification of target-specific, ligation-dependent circular probe. Gene 211:277-285.

Received 11 December 2000; accepted 5 June 2001.

Address correspondence to:

Dr. Phil Giffard

CRC for Diagnostics

QUT Gardens Point Campus

GPO Box 2434

Q 4001, Australia

e-mail:p.giffard@qut.edu.au.

For reprints of this or any other article, contact Reprints@BioTechniques.com 